\title{
IgE-mediated 15-hydroxyeicosatetraenoic acid (15-HETE) generation by peripheral blood leukocytes: its association with basophil activation
}

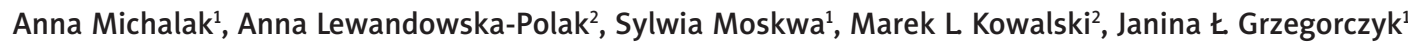 \\ ${ }^{1}$ Department of Microbiology and Medical Laboratory Immunology, Medical University of Lodz, Lodz, Poland \\ Head of the Department: Prof. Janina Ł. Grzegorczyk PhD \\ 2Department of Immunology, Rheumatology and Allergy, Medical University of Lodz, Lodz, Poland \\ Head of the Department: Prof. Marek L. Kowalski MD, PhD
}

Postep Derm Alergol 2015; XXXII (4): 262-267

DOI: $10.5114 /$ pdia.2015.52741

\begin{abstract}
Introduction: Allergen-induced basophil activation has been associated with the release of several mediators and with an increased expression of CD203c molecules on basophils.

Aim: To assess the influence of specific allergens on the generation of 15-hydroxyeicosatetraenoic (15-HETE) from peripheral blood leukocytes in relation to basophil activation, on the basis of CD203c molecule expression and histamine release.

Material and methods: The study included 15 patients with clinical symptoms of birch pollen allergy confirmed by a positive skin prick test with the birch allergen, and 6 healthy controls. Leukocytes isolated from peripheral blood were incubated with 3 concentrations of the birch pollen allergen (Bet $v$ 1), anti-lgE or with ionophore A23187.

Results: In vitro challenge of leukocytes from allergic patients with $1 \mathrm{ng} / \mathrm{ml}$ of allergen induced a significant increase in 15 -HETE generation. An increase above $30 \%$ was observed in almost half the allergic patients, with mean values ranging from $40 \%$ to $46 \%$, but not in healthy controls. Anti-IgE antibodies increased 15 -HETE generation in 5 patients (termed IgE+), and the allergen induced a significant increase in 15-HETE in all patients who reacted to anti-lgE. The mean CD203c expression on basophils of the allergic patients increased after allergen challenge, but a significant increase (> 30\%) was observed only in patients who demonstrated an increased expression after anti-lgE exposure. A significant correlation was seen between 15-HETE generation and histamine release induced by the highest concentration of the allergen $(r=0.95 ; p<0.01)$.

Conclusions: Allergen-induced, IgE-mediated activation of basophils is associated with a significant increase in 15-HETE generation.
\end{abstract}

Key words: basophil activation, birch allergy, CD203c, 15-hydroxyeicosatetraenoic

\section{Introduction}

Allergy to birch pollen, clinically manifesting as rhinitis, conjunctivitis or bronchial asthma, is associated with the immunoglobulin E (IgE)-related inflammatory process. Allergen-induced bridging of the IgE antibodies, followed by their binding to high affinity FcERI receptors on mast cells and basophils, results in cell activation and the release of several preformed and newly-synthesized mediators which induce allergy symptoms [1]. Basophils and mast cells act not only as effector cells during the early phase of the allergic reaction, but also contribute to the development of chronic allergic inflammation [1-3].
The activation of basophils and mast cells during hypersensitivity reactions is associated with the enhancement of the arachidonic acid metabolism and the generation of cyclooxygenase (prostaglandins and thromboxanes) and 5-lipoxygenase (cysteinyl leukotrienes) metabolites [3].

15-lipooxygenase (15-LO) is an enzyme which metabolizes arachidonic acid to 15 -hydroxyeicosatetraenoic acid (15-HETE), which is a chemically active metabolite characterized by a short half-life. It has been documented that unstimulated peripheral blood leukocytes produce 15-HETE, and this production may be enhanced by

Address for correspondence: Anna Michalak, Department of Microbiology and Medical Laboratory Immunology,

Medical University of Lodz, 251 Pomorska St, $92-213$ Lodz, Poland, phone: +48 606718 956, e-mail: anna.michalak@umed.lodz.pl Received: 21.01.2015, accepted: 28.02.2015. 
aspirin in aspirin-hypersensitive, but not in aspirin-tolerant, patients [4]. It has not been assessed whether allergen-included basophil activation is associated with the release of 15 -HETE.

Traditionally, activation of basophils by the allergen in vitro has been assessed by measurement of histamine release, which is a highly-specific marker of activation [5]. More recently, measurement of the expression of CD63 and CD203c molecules on the surface of leukocytes has been used to assess basophil activation [6]. The CD203c molecule is expressed on the surface of basophils, mast cells and their precursor CD34+ cells, and its expression is rapidly increased after the cells have been activated [7-10].

\section{Aim}

The aim of the study was to assess the influence of specific allergens on the generation of 15-hydroxyeicosatetraenoic (15-HETE) from peripheral blood leukocytes in relation to basophil activation, on the basis of CD203c molecule expression and histamine release.

\section{Material and methods}

The study included 15 patients allergic to birch pollen: 9 females aged $22-60$ years (mean age $34.6 \pm 4.7$ ) and 6 males aged $23-42$ years (mean age $25.4 \pm 3.8$ ). All the patients were diagnosed with seasonal rhinitis and conjunctivitis. One patient was diagnosed with atopic bronchial asthma. Birch pollen allergy was confirmed with a positive skin prick test and conjunctival provocation test with Bet $v 1$ allergen. The study was carried out before the birch pollen season, and at the time of the study, the patients were not taking any antihistamine drugs or nasal glucocorticosteroids. The control group consisted of 6 healthy subjects with negative skin prick tests to airborne allergens: 2 females, aged $28-58$ years (mean: $43.0 \pm 15.0$ ) and 4 males, aged $22-34$ years (mean: 27.6 \pm 2.31 years)

Leukocytes were isolated from $9 \mathrm{ml}$ of peripheral blood in anti-coagulant EDTA according to the protocol by Benveniste [11]. The blood sample was centrifuged for $5 \mathrm{~min}$ at $200 \times$ gat $4^{\circ} \mathrm{C}$. The plasma was collected into a separate tube and centrifuged for $10 \mathrm{~min}$ at $500 \times$ gat $4^{\circ} \mathrm{C}$. After removal of plasma, commercial reagent kit (Uti - Lyse ${ }^{\mathrm{TM}}$, Erythrocyte - Lysing Reagent, Dako Glostrup, Denmark) was used for lysis of erythrocytes from obtained sediment, cell pellet was resuspended in $900 \mu$ l of cold PBS (buffered saline with $\mathrm{Ca}^{2+}$ and $\mathrm{Mg}^{2+}$, Biomed Lublin). After isolation, the cells were incubated for $45 \mathrm{~min}$ at $37^{\circ} \mathrm{C}$ with anti-lgE (Monoclonal Antibody Anti-Human IgE (De2), Beckman Coulter), ionophore A23187 (Calcium ionophore, Calbiochem, A23187, Merc) or with Bet $v 1$ allergen at 3 concentrations: 1,10 or $100 \mathrm{ng} / \mathrm{ml}$. After centrifuging the samples for $5 \mathrm{~min}\left(200 \times \mathrm{g}\right.$, at $\left.4^{\circ} \mathrm{C}\right)$, the supernatant was separated from the cell precipitate and stored at $-20^{\circ} \mathrm{C}$. The hista- mine and 15-HETE concentrations were measured by ELISA (Assay Designs, Inc. Hines Drive Ann Arbor, MI 48108). The histamine test was considered significant for values more than $60 \%$ above baseline, 15-HETE increase was considered significant at over 30\% above the baseline and an increase in G-mean was also considered significant when $30 \%$ above the baseline.

The expression of CD203c molecules, expressed as the G-mean fluorescence, was evaluated with flow cytometry, using Flow Max software. In brief, cells were incubated with $100 \mu \mathrm{l}$ of cold buffered saline with $\mathrm{Ca}^{2+}$ and $\mathrm{Mg}^{2+}$ ions (Biomed Lublin) and $20 \mu \mathrm{l}$ of anti-CD203C monoclonal antibodies stained with phycoerythrin (PE-Rycoer-phycoerythrin) (CD203C-PE, PN IM3575, 97A6, Immunotech). Next, the samples were incubated for $60 \mathrm{~min}$ at $4^{\circ} \mathrm{C}$ and then analyzed by flow cytometry: at least 50,000 cells were analyzed. Based on reported methodology, a 30\% increase in CD203c molecule count over non-stimulated controls was considered to be significant $[10,12]$.

\section{Statistical analysis}

The Mann-Whitney $U$ test was applied to analyze the differences between groups. The Spearman's rank correlation coefficient was used to evaluate the relationship between CD203c and 15-HETE production, and the release of histamine. All statistical analyses were performed with the Statistica software package. The level of statistical significance was $p \leq 0.05$.

\section{Results}

\section{Release of 15-HETE from leukocytes.}

The stimulation of leukocytes with Bet $v 1$ allergen increased the mean concentration of 15-HETE in leukocyte supernatants from all allergic patients, but the increase was significant only for the samples subjected to $1 \mathrm{ng} /$ $\mathrm{ml}$ of allergen: from a mean value of $6.93 \pm 1.55 \mathrm{ng} / \mathrm{ml}$ to $8.32 \pm 1.80 \mathrm{ng} / \mathrm{ml}(p=0.03)$ (Figure 1$)$. An increase in 15-HETE generation of more than $30 \%$ was observed in almost half the patients: 7 of them (46.7\%) after stimulation with $1 \mathrm{ng} / \mathrm{ml}$ of allergen, 6 (40\%) after stimulation with $10 \mathrm{ng} / \mathrm{ml}$ of allergen, and 7 (46.7\%) after stimulation with $100 \mathrm{ng} / \mathrm{ml}$ of allergen. After cell stimulation with anti-lgE, the mean level of 15-HETE significantly increased from a mean value of $6.93 \pm 1.55 \mathrm{ng} / \mathrm{ml}$ to $9.60 \pm 3.70 \mathrm{ng} / \mathrm{ml}(p<0.01)$. An increase greater than $30 \%$ was observed in 5 of the 15 (33.3\%) patients and these patients have been defined as the anti-IgE responders (anti-lgE(+)), while the remaining patients assigned as anti-lgE non-responders (anti-lgE(-)). Retrospective analysis revealed that all anti-IgE(+) patients released 15 -HETE in response to the allergen at all three concentrations: The 15-HETE concentration increased from baseline $4.21 \pm 2.87 \mathrm{ng} / \mathrm{ml}$ to 11.18 


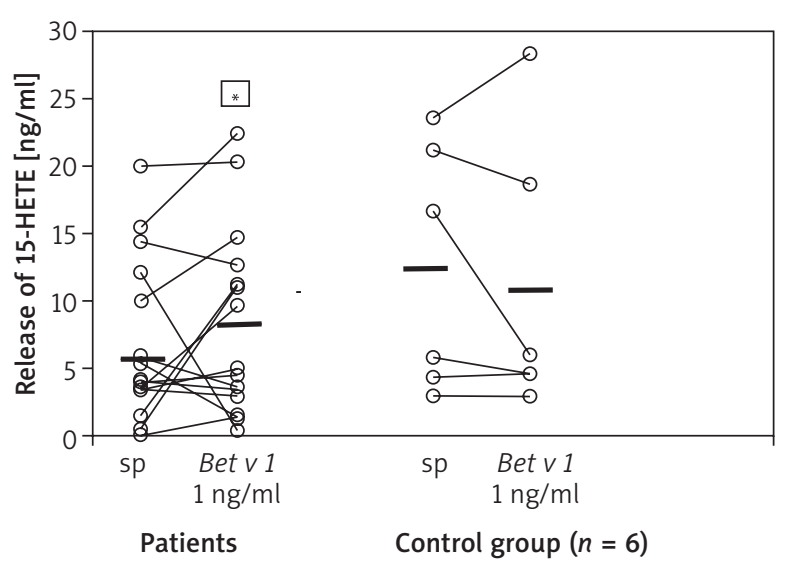

Figure 1. The release of 15 -HETE acid in patients after stimulation with birch allergen Bet $v 1\left(^{*} p=0.03\right)$

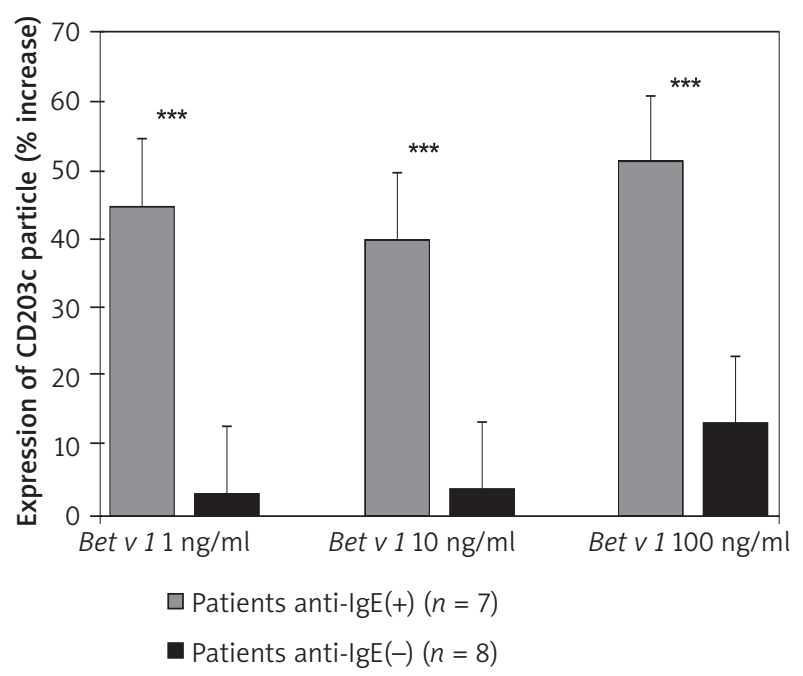

Figure 3. Allergen-induced increase in CD203c molecule expression in anti-lgE $(+)$ and anti-lgE $(-)$ patients $\left({ }^{* *} p<0.01\right)$

$\pm 3.35 \mathrm{ng} / \mathrm{ml}$ after $1 \mathrm{ng}$ of allergen, $10.55 \pm 3.70 \mathrm{ng} / \mathrm{ml}$ after $10 \mathrm{ng}$ of allergen and $7.77 \pm 4.09 \mathrm{ng} / \mathrm{ml}$ after $100 \mathrm{ng}$ of allergen (Figure 2). No change in mean 15-HETE generation was noticed in anti-lgE(-) patients after allergen challenge. In the group of healthy subjects, neither the allergen at any of the three different concentrations, nor the anti-lgE induced any significant increase in mean 15-HETE generation.

\section{Expression of CD 203c molecule on basophils}

As compared to unstimulated cells (G-mean value: $15.33 \pm 0.87$ ) all three concentrations of the allergen induced significant increases in CD203c expression: 18.25 \pm 1.09 ( $p<0.01)$ for $1 \mathrm{ng} / \mathrm{ml} ; 17.73 \pm 1.10(p=0.02)$ for $10 \mathrm{ng} / \mathrm{ml}$ and $19.29 \pm 0.97(p<0.01)$ for $100 \mathrm{ng} / \mathrm{ml}$. An

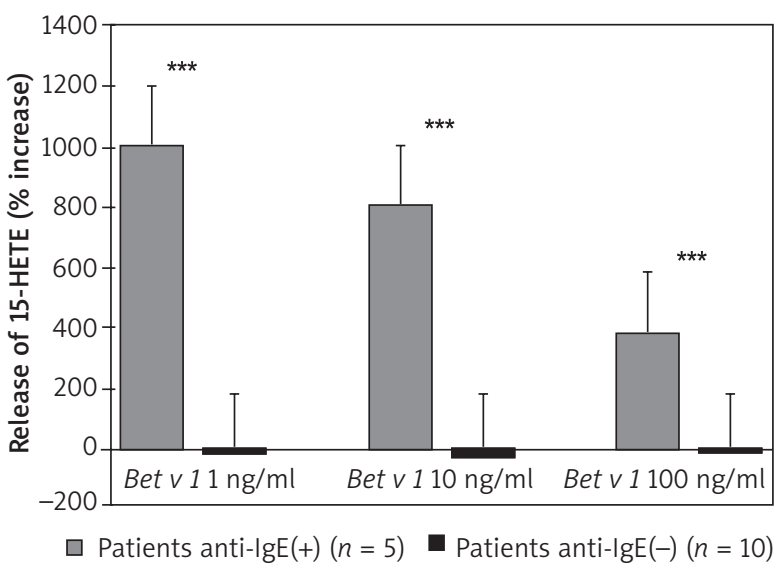

Figure 2. Allergen-induced increase in 15-HETE generation in anti-lgE(+) and anti-lgE(-) patients $\left({ }^{* *} p<0.01\right)$

increase greater than 30\%, which was considered significant, was observed in 7 (46.7\%) of 15 patients after the stimulation of cells with $1 \mathrm{ng} / \mathrm{ml}$ of allergen, in 6 of $15(40 \%)$ patients after $10 \mathrm{ng} / \mathrm{ml}$ of allergen and in 8 of 15 (53.3\%) patients after $100 \mathrm{ng} / \mathrm{ml}$ of allergen. However, in samples from the control group of non-allergic subjects, the incubation of leukocytes with allergen did not affect the mean expression of CD203c marker (Figure 3).

Cell stimulation with anti-lgE resulted in a significant increase in G-mean, above 30\% from baseline, in 7 of 15 (44.7\%) subjects. All anti-lgE(+) patients demonstrated an increase in expression of $\mathrm{G}=$ mean greater than $30 \%$ in response to all three allergen concentrations: in anti-lgE(-) patients, an increase inCD203 G-mean was observed only after the highest allergen concentration (Figure 3).

An increase in G-mean by more than $30 \%$ was also observed in 2 of 6 (33\%) healthy respondents after stimulation with anti-IgE. These subjects failed to show any increase in CD203c after allergen exposure.

\section{Histamine release from basophils}

In allergic patients, the release of histamine significantly increased from baseline, $4.75 \pm 1.11 \mathrm{ng} / \mathrm{ml}$, to 30.77 $\pm 8.38(p<0.01)$ after exposure to $1 \mathrm{ng} / \mathrm{ml}$ of allergen, to $25.06 \pm 8.04(p<0.01)$ after exposure to $10 \mathrm{ng} / \mathrm{ml}$ of allergen and to $35.94 \pm 10.66(p<0.01)$ after exposure to $100 \mathrm{ng} / \mathrm{ml}$ of allergen. A significant increase in histamine release, over $60 \%$, was observed in 12 of the 15 (75\%) patients after application of $1 \mathrm{ng} / \mathrm{ml}$ of allergen, in $11(73.3 \%)$ patients after $10 \mathrm{ng} / \mathrm{ml}$ of allergen and in $13(86.6 \%)$ patients after $100 \mathrm{ng} / \mathrm{ml}$ of allergen. All but 1 patient demonstrated a significant release of histamine in response to anti-lgE.

All anti-lgE(+) patients demonstrated a release of histamine $60 \%$ greater than the mean baseline value, 4.75 


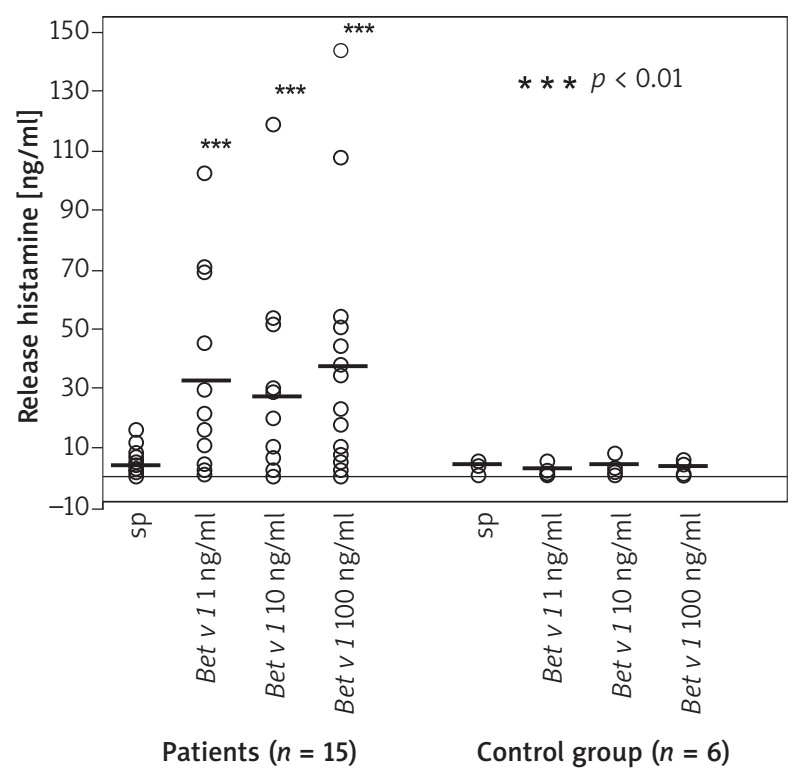

Figure 4. Allergen-induced histamine release in allergic patients and healthy controls

$\pm 1.11 \mathrm{ng} / \mathrm{ml}$, in response to the allergen at all three concentrations: to $30.77 \pm 8.62 \mathrm{ng} / \mathrm{ml}$ after $1 \mathrm{ng} / \mathrm{ml}$ of allergen, to $25.06 \pm 8.04 \mathrm{ng} / \mathrm{nl}$ after $10 \mathrm{ng} / \mathrm{ml}$ of allergen and to $35.94 \pm 10.66 \mathrm{ng} / \mathrm{ml}$ after $100 \mathrm{ng} / \mathrm{ml}$ of allergen. The anti-lgE(-) patient did not demonstrate any histamine release after allergen challenge. Stimulation of leukocytes from the healthy subjects with the birch allergen did not affect histamine release (Figure 4).

\section{Correlation between 15-HETE generation and basophil activation markers}

A significant correlation was observed between 15 -HETE production and histamine release induced by the highest concentration of the allergen $(r=0.95$; $p<0.01$ ) (Figure 5). There was a modest correlation between CD203C expression and histamine release after stimulation with the allergen $(r=0.22 ; p=0.03)$. No correlation was seen between allergen-induced 15-HETE production and CD203C expression ( $r=-0.004 ; p=0.09)$.

\section{Discussion}

This is the first study to demonstrate that peripheral blood leukocytes, presumably basophils, from allergic patients generate 15-HETE when activated with a specific allergen. It is assumed that in this model, the allergen activates basophils by bridging specific IgE molecules on the surface of the cells, which triggers IgE-mediated cell activation typical of the allergic reaction. Accordingly, no increase was observed in the production of 15-HETE after allergen exposure in the control group of non-allergic subjects. As the study was performed on a hetero-

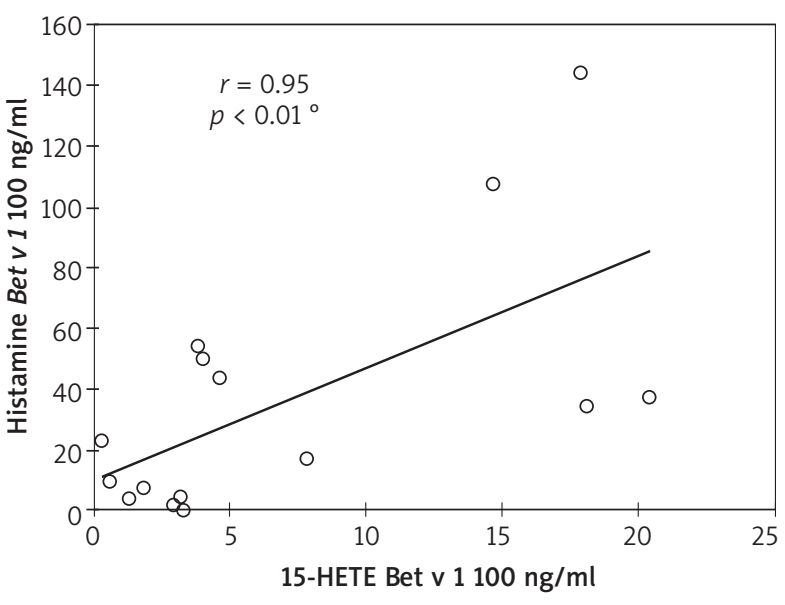

Figure 5. Correlation between allergen-induced increase in $15-$ HETE generation and release of histamine $(100 \mathrm{ng} / \mathrm{ml}$ allergen concentration)

geneous population of cells, it cannot be excluded that other inflammatory cells were activated by the allergen: 15-HETE can be generated both by mononuclear cells and granulocytes. However, the strong correlation observed between the production of 15-HETE and increased release of histamine $(r=0.95)$, which is the most specific activation marker for basophils, implied that 15-HETE originated from the IgE-activated basophils. The increased synthesis of 15-HETE seen after cell incubation with anti-lgE, which activates basophils by linking IgE molecules bound with Fce receptors, regardless of the specificity of the IgE molecules, further evidences that the mechanism of 15-HETE generation is IgE dependent.

The peripheral blood leukocytes from the subjects allergic to birch pollen demonstrated significant individual differences regarding the release of 15-HETE, and a specific allergen induced a significant increase in 15-HETE production only in approximately half the patients. Such variability might result from the individual "releasability" of basophils. This releasability of basophils depends primarily on the number of IgE molecules bound to the Fc receptors on the basophils, but also on intrinsic intercellular biochemical mechanisms related to Fc receptor signaling pathways which allow "releasers" to be distinguished from "non-releasers" [12].

Both inpatients from "responders" and "non-responders" groups, blood samples were collected from September to December, between the tree pollen seasons in Poland, when the birch pollen allergen Bet $v 1$ is absent in the atmosphere.

Significant generation of 15-HETE was detected in only half the patients after in vitro challenge of leukocytes with anti-lgE. These patients were termed algE(+). Retrospective analysis revealed that all anti-lgE(+) patients released 15 -HETE in response to all three allergen concentrations, while anti-lgE(-) patients did not 
show any increase in 15-HETE. This observation further confirms that the mechanisms of allergen-induced 15-HETE generation from basophils in allergic patients are dependent on IgE. There was a full pool of leukocytes varying in terms of quality isolated in our study, which was a potential source of 15-HETE in the tested samples. In the course of an allergic reaction, mediators are released from granules. At the same time cellular enzymes may be released. They can potentially modulate the activation of the cells, similarly to histamine it self, which if released as a feedback path, may affect the arachidonic acid cascade. Histamine may also modulate the expression of COX cyclooxygenase pathway which is associated with the release of 15-HETE. This mechanism has not been elucidated and require further research related to the use of COX inhibitor (i.e. Celecoxib).

Similar heterogeneity was observed in response to allergen exposure when flow cytometry was used to measure basophil activation according to membrane expression of CD203c $[13,14]$. In this study, CD203c expression, expressed as the geometric mean of fluorescence intensity (G-mean), was shown to correlate with the percentage of cells demonstrating CD203c expression [15-17]. Again, CD203c expression was found to be enhanced by allergen stimulation in only about half the patients, and anti-lgE(+) patients demonstrated a significantly stronger response to the allergen than anti-lgE(-) patients. Histamine release appeared to be more sensitive than the first two cell activation markers (15-HETE and CD 203c): at the maximum concentration of the allergen, almost $90 \%$ of the patients demonstrated a significant increase in histamine release. A strong correlation was seen between increased 15-HETE generation and histamine release from basophils, strongly implicating basophils as a cellular source of 15-HETE.

Lipid mediators, specifically cysteinyl leukotrienes and prostaglandins, are important mediators of IgE mediated allergic reactions and have been also associated with allergen-induced basophil activation [18-21]. 15-HETE, which is generated from arachidonic acid, predominantly via the 15-lipoxygenase pathway, is a potent mediator of inflammation with several pro-allergic activities, which include smooth muscle constriction, increased microvascular permeability, mucus secretion, and leukocyte chemotaxis [22]. However, very little information is available on the role of 15-lipoixygenase metabolites in allergic reactions and basophil activation. IgE dependent release of 15-HETE was demonstrated originally in dog mastocytoma cells, but no other study has addressed this phenomenon since then Phillips et al. [23]. Expression of 15-LO may be increased in the BAL of patients with atopic asthma and in the airway mucosa of patients with severe asthma [24, 25]. A more recent study demonstrates that 15 -HETE is released in the tear fluid of pollen-allergic patients, but no consistent increase in generation of 15-HETE was observed following local chal- lenge with allergen [26]. Our current study indicates that 15-HETE may be released from basophils, and possibly mast cells, following stimulation with a specific allergen, and should be reconsidered as a potential mediator of lgE-mediated allergic reactions.

In conclusion, our study demonstrates that a significant increase in the release of 15-HETE occurs from leukocytes enriched with a basophil fraction after allergen stimulation. A highly significant correlation found between the release of histamine and the generation of 15-HETE after allergen stimulation suggests that 15-HETE plays a possible role in the immediate allergenic reaction. The mechanism of acid release 15 -HETE from peripheral blood leukocytes after induction of a specific allergen has not been elucidated so far. Also, there are no research data on the real source of the release of this mediator after induction with a specific allergen. The 15-HETE probably is released de novo from phospholipid membranes after activation of specific enzymes in the cell [27, 28].

The release of 15-HETE in high concentrations from leukocytes after induction of a specific allergen Bet $v 1$ in vivo is responsible for the dynamics of the inflammatory reaction. A high concentration of this mediator is responsible for the inhibition of 5-LOX and the 5-lipoxygenase participates in the formation of leukotrienes LTC4 and LTB4 from arachidonic acid. The strongest pro-inflammatory effect is characteristic of leukotrienes derived from arachidonic acid. Leukotriene B4 is the most active factor which modulates the chemotaxis of neutrophils. Leukotrienes C4, D4, E4 are mediators of inflammation and are involved in allergic process. If these mechanisms are suppressed the consequence is the increased development of inflammation in the upper respiratory tract.

Other researchers [29] argue that mediators synthesized by the cyclooxygenase pathway can potentially affect the course and severity of the inflammatory response in the airways. They confirm that the long-term mediator stimulation may lead to structural changes in the epithelial cells, which is reflected in the airway remodeling.

\section{Conclusions}

These results suggest that 15 -HETE, elicited by the IgE-dependent pathway and generated by basophils, may play a role as a mediator of allergic reactions. This observation should prompt further studies on the role of 15-lipooxygenase pathways in IgE-mediated allergic reactions.

\section{Conflict of interest}

The authors declare no conflicts of interest.

\section{References}

1. Obata K, Mukai K, Tsujimura Y, et al. Basophils are essential initiators of a novel type of chronic allergic inflammation. Blood 2007; 110: 913-20. 
2. Mukai K, Obata K, Tsujimura Y, et al. New insights into the roles for basophils in acute and chronic allergy. Allergol Int 2009; 58: 11-9.

3. Gibbs BF. Human basophils as effectors and immunomodulators of allergic inflammation and innate immunity. Clin Exp Med 2005; 5: 43-9.

4. Kowalski ML, Ptasinska A, Bienkiewicz B, et al. Differential effects of aspirin and misoprostol on 15-hydroxyeicosatetraenoic acid generation by leukocytes from aspirin-sensitive asthmatic patients. J Allergy Clin Immunol 2003; 112: 505-12.

5. Novak N, Mete N, Bussmann C, et al. Early suppression of basophil activation during allergen-specific immunotherapy by histamine receptor 2. J Allergy Clin Immunol 2012; 130: 1153-8.

6. Crockard AD, Ennis M. Basophil histamine release tests in the diagnosis of allergy and asthma. Clin Exp Allergy 2001; 3: 345-50.

7. Goding JW. Ecto-enzymes: physiology meets pathology. J Leukoc Biol 2000; 67: 285-311.

8. Potapińska O, Górska E, Zawadzka-Krajewska A, et al. The usefulness of CD203c expression measurement on basophils after activation with grass pollen and Dermatophagoides pteronyssinus antigens. Preliminary study. Pneumonol Alergol Pol 2009; 77: 138-44.

9. Hauswirth AW, Natter S, Ghannadan M, et al. Recombinant allergens promote expression of CD203c on basophils in sensitized individuals. J Allergy Clin Immunol 2002; 110: 102-9.

10. Bühring HJ, Streble A, Valent $P$, et al. The basophil-specific ectoenzyme E-NPP3 (CD203c) as a marker for cell activation and allergy diagnosis. Int Arch Allergy Immunol 2004; 133: 317-29.

11. Benveniste J. The human basophil degranulation test as an in vitro method for the diagnosis of allergies. Clin Exp Allergy 1981; 11: 1-11.

12. Vonakis BM, Saini SS. Syk-deficient basophils from donors with chronic idiopathic urticaria exhibit a spectrum of releasability. J Investig Allergol Clin Immunol 2008; 121: 262-4.

13. Wedemeyer J, Tsai M, Galli SJ. Roles of mast cells and basophils in innate and acquired immunity. Curr Opin Immunol 2000; 12: 624-31.

14. Ebo DG, Bridts CH, Hagendorens MM, et al. Basophil activation test by flow cytometry: present and future applications in allergology. Cytometry B Clin Cytom 2008; 74: 201-10.

15. Green DR, Cotter TG. Introduction: apoptosis in the immune system. Semin Immunol 1992; 4: 355-62.

16. Belon P, Cumps J, Ennis M, et al. Histamine dilutions modulate basophil activation. Inflamm Res 2004; 53: 181-8.

17. Ebo DG, Bridts $\mathrm{CH}$, Mertens $\mathrm{CH}$. Analyzing histamine release by flow cytometry (HistaFlow): a novel instrument to study the degranulation patterns of basophils. I Immunol Methods 2012; 375: 30-8

18. Lie WJ, Homburg CH, Kuijpers TW, et al. Regulation and kinetics of platelet-activating factor and leukotriene C4 synthesis by activated human basophils. Clin Exp Allergy 2003; 33: 1125-34.

19. Ugajin T, Satoh T, Kanamori T, et al. FcepsilonRI, but not FcgammaR, signals induce prostaglandin D2 and E2 production frombasophils. Am J Pathol 2011; 179: 775-82.

20. de Weck AL, Sanz ML Cellular allergen stimulation test (CAST) 2003 - a review. I Investig Allergol Clin Immunol 2004; 14: 253-73.

21. Furukawa K, Tengler R, de Weck AL, et al. Simplified sulfidoleukotriene ELISA using LTD4-conjugated phosphatase for the study of allergen-induced leukotriene generation by isolated mononuclear cells and diluted whole blood. J Investig Allergol Clin Immunol 1994; 4: 110-5.

22. Narayanankutty A, Reséndiz-Hernández JM, Falfán-Valencia R, et al. Biochemical pathogenesis of aspirin exacerbated respiratory disease (AERD). Clin Biochem 2013; 46: 566-78.

23. Phillips MJ, Gold WM, Goetzl EJ. IgE-dependent and ionophore-induced generation of leukotrienes by dog mastocytoma cells. J Immunol 1983; 131: 906-10.

24. Kuitert LM, Newton R, Barnes NC. Eicosanoid mediator expression in mononuclear and polymorphonuclear cells in normal subjects and patients with atopic asthma and cystic fibrosis. Thorax 1996; 51: 1223-8.

25. Chu HW, Balzar S, Westcott JY. Expression and activation of 15-lipoxygenase pathway in severe asthma: relationship to eosinophilic phenotype and collagen deposition. Clin Exp Allergy 2002; 32: 1558-65.

26. Jedrzejczak-Czechowicz M, Lewandowska-Polak A, Jarzebska M, Kowalski ML. Mast cell and eosinophil activation during early phase of grass pollen-induced ocular allergic reaction. Allergy Asthma Proc 2011; 32: 43-8.

27. Demoly P, Lebel B, Arnoux B. Allergen-induced mediator release tests. Allergy 2003; 58: 553-8.

28. Norris PC, Gosselin D, Reichart D, et al. Phospholipase A2 regulates eicosanoid class switching during inflammasome activation. Proc Natl Acad Sci U S A 2014; 111: 12746-51.

29. Jakiela B, Gielicz A, Plutecka H, et al. Eicosanoid biosynthesis during mucociliary and mucous metaplastic differentiation of bronchial epithelial cells. Prostaglandins Other Lipid Mediat 2013; 106: 116-23. 